\title{
ARCHIV DER PHARMACIR.
}

CXXXVII. Bandes zweites Heft.

\section{Erste Abtheilung. \\ I. Physik, Chemie und praktische Pharmacie.}

\section{Chemische Beobachtungen; ron}

Ed. H a rms.

In Bezug auf den Vorgang, welcher bei der Zersetzung des Bromschwefels durch Wasser statt findet, scheint es, als wenn hierbei auch schweflige Säure auftritt. Ist dieses nun der Fall, so muss durch wechselseitige Zersetzung derselben mit dem Schwefelwasserstoff neben Schwefel, der sich ausscheidet, Pentathionsäure entstehen. Ausserdem aber wird der Bromschwefel auf die schweflige Säure reagiren in der Weise, dass Tri- und Tetrathionsäure (vielleicht ursprünglich Pentathionsäure) sich bilden. Oder sollte sich der Bromschwefel anders verhalten, als der Chlorschwefel? Ich habe angeführt, dass auf Zusatz von Brom zu dem Säuregemisch Schwefel gefallt werde. Dieser Schwefel rülhrt offenbar von den Thionsäuren her. Versetzt man Pentathionsäure mit geringen Mengen von Brom, so entsteht je nach der Concentration ein mehr oder minder starker Niederschlag, der auf weiteren Zusatz von Brom nach und nach verschwindet :

$$
\mathrm{S}^{5} \mathrm{O}^{5}+\mathrm{Br}+\mathrm{HO}=2 \mathrm{SO}^{3}+\mathrm{HBr}+3 \mathrm{~S}
$$

ferner $3 \mathrm{~S}+9 \mathrm{Br}+9 \mathrm{HO}=3 \mathrm{SO}^{3}+9 \mathrm{HBr}$. 
Man muss voraussetzen, dass bei Ausschluss des Wassers eine Verbindung entstehen würde, die an Stelle von Schwefel Brom enthält. Denn alle Reactionen scheinen darauf hinzuweisen, dass die Thionsäuren überhaupt rationell als Schwefelsäuren betrachtet werden müssen, in welchen ein Theil des Sauerstoffs durch Schwefel vertreten ist. Diese Ansicht über die Constitution der Thionsäuren' ist nicht neu; schon vor ein Paar Jahren lehrte Prof. Koene wenn ich nicht irre - die dithionige Säure als $S\left(O^{2} S\right)$ betrachten und stellte sie dadurch also der gewöhnlichen Schwefelsäure an die Seite. Und in der That möchte eine andere Anschauung kaum zulässig sein. Die folgenden Beispiele erklären sich von selbst:

$$
\mathrm{AgO}, \mathrm{S}\left(\mathrm{O}^{2} \mathrm{~S}\right)=\mathrm{AgS}+\mathrm{S}\left(\mathrm{O}^{2} \mathrm{O}\right)
$$

$\mathrm{As} \mathrm{O}^{5}+5\left[\mathrm{NaO}, \mathrm{S}\left(\mathrm{O}^{2} \mathrm{~S}\right)\right]=\mathrm{As} \mathrm{S}^{5}+5\left[\mathrm{NaO}, \mathrm{S}\left(\mathrm{O}^{2} \mathrm{O}\right)\right]$ $\mathrm{Hg}^{2} \mathrm{O}, \mathrm{NO}^{5}+\mathrm{NaO}, \mathrm{S}\left(\mathrm{O}^{2} \mathrm{~S}\right)=\mathrm{Hg}^{2} \mathrm{~S}+\mathrm{NaO}, \mathrm{S}\left(\mathrm{O}^{2} \mathrm{O}\right)$ etc.

Wie bei der Einwirkung von Sauerstoff auf schwefligsaures Kali $\mathrm{KO}, \mathrm{SO}^{3}$ entsteht, so bildet sich bei der Einwirkung von Schwefel auf das nämliche Salz KO, $\mathrm{S}\left(\mathrm{O}^{2} \mathrm{~S}\right)$. Derselbe Vorgang findet statt. Ferner hat man für

$$
\mathrm{MgO}, \mathrm{S}\left(\mathrm{O}^{2} \mathrm{~S}\right)+\mathrm{KO}, \mathrm{S}\left(\mathrm{O}^{2} \mathrm{~S}\right)+6 \mathrm{aq}
$$

und $\mathrm{MgO}, \mathrm{S}\left(\mathrm{O}^{2} \mathrm{~S}\right)+\mathrm{AmO}, \mathrm{S}\left(\mathrm{O}^{2} \mathrm{~S}\right)+6 \mathrm{aq}$

die entsprechenden Schwefelsäuresalze, welche nicht allein in der Zusammensetzung, sondern auch in manchen Eigenschaften mit einander übereinstimmen. Und ist $\mathrm{S}\left(\mathrm{O}^{2} \mathrm{Cl}\right)$ nicht eine analoge Verbindung? Man hat sie zwar auch als $\mathrm{SCl}^{3}+2 \mathrm{SO}^{3}$ aufgefasst, aber nie als (SCl) $\mathrm{O}^{2}$. Ueberhaupt kommen Substitutionen des Sauerstoffs durch Schwefel häufig vor, und dürfte ich in dieser Beziehung vorzugsweise an die $\mathrm{P}\left(\mathrm{O}^{3} \mathrm{~S}^{2}\right)$ erinnern. Ist nun die unterschweflige Säure nach der Formel $\mathrm{S}\left(\mathrm{O}^{2} \mathrm{~S}\right)$ zusammengesetzt, so entwickelt sich hieraus von selbst, dass die Constitution der Tetrathionsäure durch die Formel $\mathrm{S}^{2}\left(\mathrm{O}^{5} \mathrm{~S}^{2}\right)$ ausgedrückt werden muss, denn:

$2 \mathrm{NaO}, \mathrm{S}\left(\mathrm{O}^{2} \mathrm{~S}\right)+\mathrm{J}=\mathrm{NaJ}+\mathrm{NaO}, \mathrm{S}^{2}\left(\mathrm{O}^{5} \mathrm{~S}^{2}\right)$

$\mathrm{Fe}^{2} \mathrm{O}^{3}, 2 \mathrm{~S}\left(\mathrm{O}^{2} \mathrm{~S}\right)=2 \mathrm{FeO}+\mathrm{S}^{2}\left(\mathrm{O}^{5} \mathrm{~S}^{2}\right)$.

Ueber die Trithionsäure spricht sich Langlois in 
folgender Weise aus: D'après sa composition, ce nouvel acide pourrait être considéré comme formé d'acide sulfurique et d'acide hyposulfureux ordinaire $=\mathrm{SO}^{3}+\mathrm{S}^{2} \mathrm{O}^{2}$, ou bien encore d'acide hyposulfurique et de soufre $=\mathrm{S}^{2} \mathrm{O}^{5}$ $+\mathrm{S}$. Ses propriétés nous déterminent à nous arrêter à cette dernière supposition et à proposer de lui donner provisoirement le nom d'acide sulfhyposulfurique et de désigner ses combinations salines par le nom de sulfhyposulfates. (Ann. de Chim. et de Phys. Sér. III. T. IV. p. 77.) Nach dem Entdecker selbst also, der sie ohne Zweifel am genauesten studirt hat, muss die Trithionsäure als $\mathbf{S}^{2} \mathrm{O}^{5}+\mathrm{S}$ betrachtet werden, und diese Formel fällt zusammen mit $\mathrm{S}^{2}\left(\mathrm{O}^{5} \mathrm{~S}\right)$.

Hiernach würden die Verbindungen des Schwefels mit Sauerstoff durch folgende Formeln repräsentirt werden müssen:

$$
\begin{aligned}
& \mathbf{S}^{2}\left(\mathrm{O}^{5} \mathrm{~S}^{3}\right)=\mathrm{S}^{5} \mathrm{O}^{5} \\
& \mathrm{~S}^{2}\left(\mathrm{O}^{5} \mathrm{~S}^{2}\right)=\mathrm{S}^{4} \mathrm{O}^{5} \\
& \mathbf{S}^{2}\left(\mathrm{O}^{5} \mathrm{~S}\right)=\mathrm{S}^{3} \mathrm{O}^{5} \\
& \mathbf{S}^{2} \mathrm{O}^{5}=\mathrm{S}^{2} \mathrm{O}^{5} \\
& \mathrm{~S}\left(\mathrm{O}^{2} \mathrm{~S}\right)=\mathrm{S}^{2} \mathrm{O}^{2} \\
& \mathrm{~S} \mathrm{O}^{3}=\mathrm{S} \mathrm{O}^{3} \\
& \mathrm{~S} \mathrm{O}^{2}=\mathrm{S} \mathrm{O}^{2} .
\end{aligned}
$$

Die von $\mathrm{Plessy}$ aufgestellten $\mathrm{S}^{5} \mathrm{O}^{7}$ und $\mathrm{S}^{6} \mathrm{O}^{7}$ existiren nach Fordos und Gélis nicht. (Ann.der Phys.u. Chem. 3. $R$. Bd. 14. p. 249.) Der Körper $\mathrm{SS}^{3} \mathrm{kann}$, wie bereits von Liebig an irgend einer Stelle niedergelegt ist, im Fünffach-Schwefclkalium angenommen werden. $\mathrm{KS}^{5}=\mathrm{KS}, \mathrm{SS}^{3}$. Die Thionsäuren haben mit einander gemein, dass sie bei der Zersetzung durch Temperaturerhöhung den Antheil des Schwefels, welcher eine elektronegative Rolle spielt, im freien Zustande abscheiden, während der von entgegengesetztem Charakter in die einfacheren Verbindungen von schwefliger Säure und Schwefelsäure übergeht. Schliesslich aber darf ich die Thatsache, dass alle unterschwefligsauren Salze eine gewisse Menge Wasser einschliessen, nicht unberührt lassen. Ana- 
Iog zusammengesetzte Körper besitzen im Allgemeinen auch analoge Eigenschaften und danach dürfte man voraussetzen, dass, wie es wasserfreie Schwefelsäuresalze giebt, auch wasserfreie dithionigsaure Salze vorkommen müssen. Dieses ist nun nicht der Fall, und man kann daraus, wenn man will, einen Grund hernehmen für die Behauptung, dass die dithionige Säure doch etwas Anderes ist, als eine substituirte Schwefelsäure. Man würde dann aber mit den Zersetzungserscheinungen, welche die Thionsäuren überhaupt und namentlich die dithionige Säure zeigen, in Verwickelung kommen.

Die Versuche von Wittstein über das Verhalten des gebrannten Kalks an der Luft (Vierteljahrsschr. Bd.V. p.60-70) erinnerten mich an eine schon vor längerer Zeit gemachte Beobachtung, die ich mir erlaube Ihnen nachträglich mitzutheilen, auf die Gefahr hin, dass sie nicht mehr neu ist. Sie betrifft die Einwirkung von Kalkhydrat auf kohlensaures Kali oder Natron. So schwach auch die Vermuthung, dass sich hiebei ein basisches Carbonat (eine Verbindung von Hydrat mit Carbonat) bilde, unterstïtzt sein mag, so erschien mir's doch nicht gänzlich unverdienstlich, sie durch eine quantitative Analyse genauer zu prüfen. Der zu dem Versuche angewandte Kalk war durch schwaches anhaltendes Glühen von reinem oxalsaurem Kalk erhalten; er enthielt im Mittel zweier vorhergegangenen Bestimmungen 13,08 Procent Carbonat, erwärmte sich übrigens beim Uebergiessen mit Wasser und zerging dabei zu einem zarten fühllosen Brei. Der so dargestellte und gelöschte Kalk wurde nun mit einem Ueberschuss von aufgelöstem kohlensaurem Kali unter Mitwirkung einer gelinden Wärme mehrere Tage lang in Berührung gelassen, die Lauge davon abgegossen und durch Auswaschen vollends entfernt. Das erhaltene Kalksalz wurde bei $40-50^{\circ} \mathrm{C}$. getrocknet und hierauf der Analyse unterworfen. 0,595 Grm. zeigten im Wöhlerschen Kohlensäure-Apparat einen Gehalt von 0,249 Grm. $\mathrm{CO}^{2}=41,85$ Proc., entsprechend 95,12 Proc. $\mathrm{CaO}, \mathrm{CO}^{2}$. 
Bei einer folgenden Bestimmung wurden 94,87 Procent $\mathrm{CaO}, \mathrm{CO}^{2}$ erhalten. Das Deficit ist zu klein, als dass man es für wesentlich halten könnte, und ich ziehe deshalb den Schluss aus diesen Zahlenresultaten, dass bei der Einwirkung von aufgelösten kohlensauren Alkalien auf Kalkhydrat das Kalkhydrat in neutralen kohlensauren Kalk verwandelt wird.

Das gewöhnliche essigsaure Bleioxyd enthält bekanntlich 3 Aeq. HO. Aus verdünntem Weingeist schiesst es nach Payen ebenfalls mit 3 Aeq. Aq. an, in der Form von Säulen des zwei- und eingliedrigen Systems; aus absolutem Alkohol kann es wasserfrei erhalten werden. Löst man eine nicht zu geringe Menge des Bleiacetats in Weingeist von 0,844 spec. Gew., und lässt ruhig erkalten, so findet keine Abscheidung von festem Salze statt, die indessen, wenn man schüttelt oder rührt, fast augenblicklich eintritt. Das so erhaltene essigsaure Bleioxyd schliesst ebenfalls 3 Aeq. HO ein.

Analyse: 0,671 Grm. verloren beim Trocknen im Luftbade 0,098 Grm., entsprechend 14,61 Proc. Wasser. Die Formel $\mathrm{PbO}, \overline{\mathrm{A}}+3$ aq verlangt 14,21 Proc.

Wegen der gleichzeitigen unvermeidlichen Entwickelung von Essigsäuredämpfen musste die Wasserbestimmung etwas zu hoch ausfallen.

\section{Ueber Tinct. Ferri acet. Rad.}

Sowohl zu Ihrer Erleichterung, als auch um mich darauf beziehen zu können, schicke ich die ursprüngliche Vorschrift zur Tinct. Ferri acet. Rad. voraus. Es sollen nämlich 2 Unzen 7 .Drachmen reines schwefelsaures Eisenoxydul und 3 Unzen reines essigsaures Bleioxyd in einem eisernen Mörser zu einem Brei zerrieben, nach Zusatz von 6 Unzen destillirten Wassers und 12 Unzen rohen Essigs in einer eisernen Schale zum Sieden erhitzt, der erkalteten Masse 10 Unzen höchst rectificirter Weingeist hinzugefügt, in einem verschlossenen Glase mehrere 
Monate lang unter öfterem Unschütteln macerirt und, nachdem die Farbe rothbraun geworden, filtrirt werden.

Die Theorie verlangt auf 3 Unzen essigsaures Bleioxyd 2 Unzen 1 Drachme 35 Gran kryst. schwetelsaures Eisenoxydul. Der Ueberschuss an letzterem Salze beträgt also 5 Drachmen 25 Gran. Dass folglich die Tinctur frei oder vielmehr so gut als frei ist von Bleioxyd, kann nicht auffallen; aber Dr. Geiseler fand auch und hat zuverlässig gezeigt, dass sie in ihrer höchsten Güte weder Schwefelsäure noch Eisenoxydul enthält. Der überschüssige Eisenvitriol muss sich mithin als solcher oder in der Form von basisch schwefelsaurem Eisenoxyd in dem Niederschlage finden. Eine andere Art der Ausscheidung ist nicht denkbar. Ferner fand Dr. Geiseler, dass die Tinctur 2,08 Proc. Eisenoxyd enthält. Prüfen wir diese Angabe durch die Rechnung. Es fallen weg aus dem oben angegebenen Satze von 33Unz.7Dr.-Gr. an überschüssigem Eisenvitriol 5 Dr.25Gr.

an Schwefelsäure. . . . $5 n 3$ n

(in $\mathbf{3} \mathbf{j} 3 \mathbf{j}$ gr. 35 Eisenvitriol)

an Bleioxyd . . . . 14,7 ,

$$
\text { Bleibt 30Unz.6Dr. 25Gr. }
$$

Hierzu der zur Oxydation des essigs.

Eisenoxyduls erforderliche Sauerstoff $-n-{ }_{n} 30$,
\[ \text { Zusammen 30Unz.6Dr.55Gr. } \]

Diese 30 Unzen 6 Drachmen 55 Gran enthalten also das Oxyd von 2 Unz. 1 Dr. 35 Gr. des gewöhnlichen schwefelsauren Eisenoxyduls $=5$ Dr. 4 Gran oder 2,05 Procent. Man sieht, dass die von Dr. Gei iseler gefundene Zahl sehr gut mit der Theorie übereinstimmt; etwas höher musste erstere ausfallen - wenn alle andern Erscheinungen, die vorausgesetzt sind, auch wirklich eintreffen - weil eben ein Verlust durch Verdunsten beim Filtriren unvermeidlich ist. Die Abänderung bei Bereitung dieser Tinctur, welche Bolle eingeführt hat, nennt Dr. Geiseler "vortrefflich", und dieses Lob verdient sie 
in der That. Dass man nicht eher und allseitig darauf gekommen ist! Aber das Nächste liegt uns oft am fernsten. Nach diesen Erörterungen gehe ich zu einem anspruchslosen Versuch über, der in der Absicht angestellt wurde, die Darstellung dieses Mittels möglichst abzukürzen und zu beschleunigen. Obgleich nun das gewünschte Resultat nicht erzielt wurde, so wird doch eine nähere Beschreibung des eingeschlagenen Verfahrens Ihnen nicht durchaus überflüssig erscheinen, zumal berücksichtigt werden dürfte, dass es einer weiteren Ausbildung und Entwickelung nicht ganz unfähig sein möchte. Lässt man nämlich die nach $R$ ad emacher's Vorschrift bereitete Tinctur kurze Zeit mit Bleihyperoxyd zusammenstehen, so findet selbst schon bei gewöhnlicher 'Temperatur eine vollkommene Oxydation des Eisensalzes statt. Die Lösung nimmt eine tiefbraune Färbung an und wird zuletzt durch Ferridcyankalium nicht mehr gebläut. Die so dargestellte Tinctur enthält indessen - wie vorauszusehen - eine grosse Menge von Bleisalz, und alle Mühe, dasselbe mittelst Schwefelsäure vollständig abzuscheiden, war vergebens. (Ueberhaupt muss der Weg, Blei aus einer Lösung von Eisenoxydsalzen vollkommen zu fällen, ohne das letztere anzugreifen, noch entdeckt werden.) Der durch Schwefelwasserstoff in der angesäuerten Tinctur bewirkte Niederschlag war stets gefürbt, bald mehr, bald weniger, zuweilen freilich sehr schwach. Unter Umständen, deren man nicht völlig Herr ist, scheint sich äthylschwefelsaures Bleioxyd zu bilden; sonst mindestens kann ich mir vorläufig die Intensitat der durch Schwefelwasserstoff hervorgerufenen Färbung, die mitunter auftrat, nicht erklären. Hierzu muss ich jedoch bemerken, dass um eine unnöthige Verdünnung der Tinctur zu vermeiden, stets eine starke Schwefelsiuure angewandt wurde. Der Gehalt an Eisenoxyd fiel unter dem richtigen Gehalt aus und wurde zu 1,78 Proc. bestimmt. Um nun noch einmal auf das von Rademacher ausgegebene Recept zurückzukommen, so hätte ebenso gut, um nicht zu sagen 
besser, statt essigsauren Bleioxyds eine äquivalente Menge essigsauren Baryts, der ja auch Handelswaare ist, vorgeschrieben werden können und die Gefahr vor einer schädlichen Verunreinigung wäre dadurch gänzlich abgewandt.

In der nach oben angegebener Methode dargestellten Tinct. Ferri acet. Rad. hatten sich einmal zarte farblose Nadeln abgeschieden, die sich bei der näheren Untersuchung als schwefelsaurer Kalk auswiesen. Der Quelle des Kalkes nachforschend, zeigte sich bald, dass die angewandte Schwefelsäure, von einem rühmlichst bekannten Handlungshause als rein erhalten, in bemerkenswerthem Grade mit Kalk oder vielmehr mit Gyps beladen war. Nach Zusatz von etwas Wasser bildeten sich in derselben nadelförmige Krystalle und bei grösserer Verdünnung trübte sich die Säure. So weit ich mich erinnere, ist diese Verunreinigung noch nicht bcobachtet worden, und wird deshalb diese Notiz nicht ohne einiges Interesse sein.

Lässt man schweflige Säure durch eine heisse Sublimatlösung streichen, so entsteht bekanntlich unter gleichzeitiger Bildung von Schwefelsäure und Chlorwasserstoffsäure ein fein krystallinischer Niederschlag von Queck. silberchlorür. Ein grosser Theil des Quecksilberchlorids entzieht sich aber der Verwandlung, selbst wenn die Flüssigkeit im Sieden unterhalten wird. Stumpft man die Säure so weit ab, dass Lackmuspapier noch entschieden geröthet wird, oder verdünnt man die Lösung bis auf das 80 fache vom Gewicht des Salzes und fährt mil dem Einleiten von schwefliger Säure fort, so schlügt sich alles oder fast alles $\mathrm{Hg} \mathrm{Cl}$ als $\mathrm{Hg}^{2} \mathrm{Cl}$ nieder. Ich versuchte nun, über stark erhitztes Sublimatpulver feuch te schweflige Säure zu leiten. Doch erhielt ich kein günstiges Resultat und die Zersetzung war sehr unvollständig. Wasserstoff schien sogar unter den nämlichen Verhält. nissen ebenso kräftig zu wirken; mindestens entwickelter sich reichliche Dämpfe von Chlorwasserstoffsäure; metal lisches Quecksilber trat hierbei nicht auf. 